\title{
A Superhelical Spiral in the Escherichia coli DNA Gyrase a C-terminal Domain Imparts Unidirectional Supercoiling Bias
}

\section{Citation}

Ruthenburg, Alexander J., Daina M. Graybosch, John C. Huetsch, and Gregory L. Verdine. 2005. "A Superhelical Spiral in the Escherichia ColiDNA Gyrase A C-Terminal Domain Imparts Unidirectional Supercoiling Bias." Journal of Biological Chemistry280 (28): 26177-84. https:// doi.org/10.1074/jbc.M502838200.

\section{Permanent link}

http://nrs.harvard.edu/urn-3:HUL.InstRepos:41511290

\section{Terms of Use}

This article was downloaded from Harvard University's DASH repository, and is made available under the terms and conditions applicable to Other Posted Material, as set forth at http:// nrs.harvard.edu/urn-3:HUL.InstRepos:dash.current.terms-of-use\#LAA

\section{Share Your Story}

The Harvard community has made this article openly available.

Please share how this access benefits you. Submit a story.

\section{Accessibility}




\title{
A Superhelical Spiral in the Escherichia coli DNA Gyrase A C-terminal Domain Imparts Unidirectional Supercoiling Bias* [
}

Received for publication, March 15, 2005, and in revised form, April 27, 2005

Published, JBC Papers in Press, May 15, 2005, DOI 10.1074/jbc.M502838200

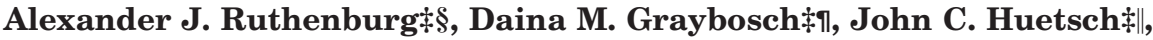 \\ and Gregory L. Verdine ${ }^{*} * *+$ \\ From the Departments of $\ddagger$ Chemistry and Chemical Biology and **Molecular and Cellular Biology, Harvard University, \\ Cambridge, Massachusetts 02138
}

\begin{abstract}
DNA gyrase is unique among type II topoisomerases in that its DNA supercoiling activity is unidirectional. The C-terminal domain of the gyrase A subunit (GyrA-CTD) is required for this supercoiling bias. We report here the x-ray structure of the Escherichia coli GyrA-CTD (Protein Data Bank code 1ZI0). The $E$. coli GyrA-CTD adopts a circular-shaped $\beta$-pinwheel fold first seen in the Borrelia burgdorferi GyrA-CTD. However, whereas the B. burgdorferi GyrA-CTD is flat, the $E$. coli GyrA-CTD is spiral. DNA relaxation assays reveal that the $E$. coli GyrA-CTD wraps DNA inducing substantial (+) superhelicity, while the B. burgdorferi GyrA-CTD introduces a more modest $(+)$ superhelicity. The observation of a superhelical spiral in the present structure and that of the Bacillus stearothermophilus ParC-CTD structure suggests unexpected similarities in substrate selectivity between gyrase and Topo IV enzymes. We propose a model wherein the right-handed $((+)$ solenoidal) wrapping of DNA around the $E$. coli GyrA-CTD enforces unidirectional (-) DNA supercoiling.
\end{abstract}

DNA topoisomerases solve the topological problems that arise in the course of normal DNA metabolism. Among the numerous functions of these essential enzymes are resolution of DNA catenation during replication and cell division and relaxation of DNA $(+)$ and $(-)$ supercoiling ${ }^{1}$ resulting from transcriptional and replicative unwinding of the genome (1). In addition to relieving torsional stress, some topoisomerases are also capable of introducing supercoils (2). In particular, bacterial DNA gyrase catalyzes the ATP-dependent introduction of

* This project was supported by National Institutes of Health Grant GM51330. The costs of publication of this article were defrayed in part by the payment of page charges. This article must therefore be hereby marked "advertisement" in accordance with 18 U.S.C. Section 1734 solely to indicate this fact.

S The on-line version of this article (available at http://www.jbc.org) contains supplemental text, references, Figs. 1-3, and a table.

The atomic coordinates and structure factors (code 1ZIO) have been deposited in the Protein Data Bank, Research Collaboratory for Structural Bioinformatics, Rutgers University, New Brunswick, NJ (http://www.rcsb.org/).

$\S$ Supported by a graduate research fellowship from the National Science Foundation.

ๆ Supported by a National Institutes of Health training grant.

\| Supported by a Robert Flack Norris undergraduate fellowship from the American Chemical Society.

+ To whom correspondence should be addressed. Tel.: 617-495-5323; Fax: 617-495-8755; E-mail: verdine@chemistry.harvard.edu.

${ }^{1}$ For our purposes, the term "supercoil" is restricted to topological changes that alter the linking number in a topologically linked DNA. The term "superhelix" refers to a physical superstructure of duplex DNA that may include both changes in writhe and twist from the relaxed state. "Solenoidal" is used as previously defined, and is interchangeable with "toroidal" $(52,53)$.
(-) supercoils (1). This activity, unique to gyrase among all topoisomerases, helps maintain prokaryotic genomes at a (-) superhelical density, which in turn is thought to lower the energy barrier for unwinding DNA during transcription and replication $(3,4)$.

Gyrase, similar to all members of the type II topoisomerase family (Topo IIs), ${ }^{2}$ catalyzes the ATP-dependent passage of one DNA duplex (T-segment) through another (G-segment), a process that requires transient introduction of a double-stranded DNA break (1). Intramolecular passage of a T-segment results in relaxation or induction of supercoils, whereas intermolecular passage results in decatenation. Most Topo IIs perform both decatenation and relaxation; yet, interestingly, gyrase exhibits an overriding preference to perform intramolecular and unidirectional $((+)$ to $(-)$ node) transfer of a T-segment through a contiguous G-segment. Gyrase is an $\mathrm{A}_{2} \mathrm{~B}_{2}$ tetramer comprising GyrA and GyrB subunits. ATPase activity resides in the Nterminal domain of GyrB (GyrB-NTD); the core cleavage-reunion complex is made up of the $\mathrm{C}$-terminal domain of GyrB (GyrB-CTD) (5) plus the N-terminal domain of GyrA (GyrANTD) $(2,6)$.

Both of the hallmark functional features unique to gyrase, its unidirectional supercoiling activity and strong preference for compact intramolecular T-segment transfer, have been attributed to the C-terminal domain of GyrA (GyrA-CTD) (7). This domain is conserved among gyrases and also members of the Topo IV family, in which it is known as ParC-CTD. Eukaryotic Topo IIs possess a CTD, but it appears to be completely different in structure and function from the prokaryotic CTDs (1, 8-10). Selective removal of the GyrA-CTD from Escherichia coli gyrase results in loss of the ability to introduce (-) supercoils in relaxed and negatively supercoiled DNA, a gain of the ability to relax (-) supercoils, and a 30-fold increase in decatenation activity (7); addition of the GyrA-CTD in trans results in restoration of the capacity to introduce $(-)$ supercoils (11, 12). Plasmid nicking/religation experiments indicate that the E. coli GyrA-CTD and full-length gyrase (in the absence of ATP) both bind DNA such that (+) superhelicity is introduced, whereas GyrB + GyrA-NTD does not alter global DNA topology upon binding $(7,13-15)$. Furthermore, $E$. coli gyrase preferentially binds and processes (+) supercoiled DNA (16), whereas GyrB + GyrA-NTD has no such preference (14). These data have been interpreted to suggest that DNA wraps around gyrase in a manner dependent upon the presence of the GyrACTD, producing (+) superhelicity, and that this wrapping plays a crucial role in controlling the directionality of supercoil in-

${ }^{2}$ The abbreviations used are: Topo, DNA topoisomerase; GyrA, gyrase A; NTD, N-terminal domain; CTD, C-terminal domain; $\beta \mathrm{ME}$, 2 -mercaptoethanol; r.m.s.d., root mean square deviation; $\beta$-CA loop, the loop containing residues 555-576. 
duction. Additional support for the notion that the full $E$. coli gyrase wraps a large continuous stretch of DNA has been provided by transient electric dichroism (17) and atomic force microscopy experiments (18); footprinting experiments have shown that gyrase protects $\sim 128$ bp of DNA, nearly all of which shows a cleavage periodicity typical for DNA bound to a surface (19-22), whereas eukaryotic Topo IIs, which lack any counterpart to the GyrA-CTD, protect only $\sim 35$ bp $(23,24)$. Together, these experiments imply a unique role for the GyrA-CTD in DNA wrapping and T-segment presentation by gyrase.

Type IV topoisomerases (Topo IVs), the other type II subclass containing a GyrA-like CTD, are unlike gyrases in that they are unable to introduce (-) supercoils in relaxed and negatively supercoiled DNA. Furthermore, Topo IV has a much higher decatenation activity than gyrase (25). Notwithstanding these differences, both classes show an overriding preference to process $(+)$ nodes $(26,27)$. This $(+)$ node preference is absent in all type II topoisomerases not belonging to the gyrase or Topo IV subclasses; these other Topo IIs relax and decatenate $(+)$ and (-) nodes at similar rates (1). Although less biochemical data are available on the ParC-CTD than the GyrA-CTD, it is reasonable to infer that the ParC-CTD also gives rise directly to the preference of Topo IV to process (+) nodes (28).

Representative structures of the GyrA-NTD (29) and the GyrB-ATPase domain from $E$. coli gyrase are known $(30,31)$, but the structure of the $E$. coli GyrA-CTD has not been available until now. The first and only structure of a GyrA-CTD, derived from the spirochete Borrelia burgdorferi, was recently published and revealed an intriguing new fold, designated a $\beta$-pinwheel (32). Here we report the structure of the $E$. coli GyrA-CTD (Fig. 1A) along with Topo I relaxation assays. Together these data form the basis of a model for how the directional bias of supercoil induction by $E$. coli gyrase is imparted by its C-terminal domain. The $E$. coli model is distinct from that proposed for B. burgdorferi, as supported by significant structural differences and divergent biochemical properties of these two GyrA-CTDs. Such differences reveal an unexpected richness of diversity in the mechanisms of DNA strand management by gyrase.

\section{EXPERIMENTAL PROCEDURES}

Protein Preparation-Plasmids encoding E. coli GyrA-CTD (532-853 or 532-841) and B. burgdorferi GyrA-CTD (501-810) were cloned from E. coli $\mathrm{K} 12$ or B. burgdorferi (American Type Culture Collection 35210D), respectively, into pET30a (Novagen) with tobacco etch virus protease-cleavable $\mathrm{N}$-terminal $\mathrm{His}_{6}$ tags and fully sequenced. Proteins were overexpressed in $E$. coli BL21(DE3)-pLysS or Rosetta2(DE3)pLysS cells at $37^{\circ} \mathrm{C}$. Lysis was accomplished by sonication in $50 \mathrm{~mm}$ sodium phosphate, $\mathrm{pH} 8.0,500 \mathrm{~mm} \mathrm{NaCl}, 15 \mathrm{~mm}$ imidazole, $10 \mathrm{~mm} \beta \mathrm{ME}$, and $1 \mathrm{~mm}$ phenylmethylsulfonyl fluoride. Both proteins were purified by nickel-nitrilotriacetic acid-agarose (Qiagen). The E. coli GyrA-CTD was further purified by a Hi-Trap heparin column (Amersham Biosciences) and eluted with $50 \mathrm{~mm}$ sodium phosphate, $\mathrm{pH} 8.0,5 \mathrm{~mm} \beta \mathrm{ME}, 1 \mathrm{~mm}$ EDTA, $10 \%$ glycerol, and 300-2000 $\mathrm{mm} \mathrm{NaCl}$. The B. burgdorferi and E. coli GyrA-CTDs for Topo I relaxation assays were finally purified by gel filtration (Superdex 200 (Amersham Biosciences) in 20 mM HEPES, $\mathrm{pH}$ 7.5, $500 \mathrm{~mm} \mathrm{NaCl}, 10 \%$ glycerol, $5 \mathrm{~mm} \beta \mathrm{ME}$, and $1 \mathrm{~mm}$ EDTA.

Structure Determination-Crystals $(\sim 300 \times 200 \times 100 \mu \mathrm{m})$ of the E. coli GyrA-CTD (532-853), with an $\mathrm{N}$-terminal $\mathrm{His}_{6}$ tag, were grown by hanging drop vapor equilibration in Nextal plates as follows: $5 \mu \mathrm{l}$ of $10-15 \mathrm{mg} / \mathrm{ml}$ protein solution (10-20 mM sodium phosphate, $\mathrm{pH} 7.4-$ 7.8, $650 \mathrm{~mm} \mathrm{NaCl}, 5 \mathrm{~mm} \beta \mathrm{ME}$, and $1 \mathrm{~mm}$ EDTA) were mixed with $5 \mu \mathrm{l}$ of well solution (100 mM Tris $\cdot \mathrm{HCl}, \mathrm{pH} 7.6-8.2$, and $1-4 \%$ polyethylene glycol 1500) and equilibrated at room temperature overnight against 1 $\mathrm{ml}$ of well solution. Extensive additive screening, removal of the $\mathrm{N}$ terminal $\mathrm{His}_{6}$ tag, and a smaller protein construct comprising residues 532-841 failed to improve the crystal quality. Crystals were cryoprotected by the stepwise addition of ethylene glycol and glycerol (to final concentrations of 20 and $8 \%$, respectively) to a polyethylene glycol 1500 -fortified mother liquor. The selenomethionine derivative of the CTD was prepared in the same strain used for the native protein production (33). In addition, $10 \mathrm{mM}$ dithiothreitol supplanted $\beta \mathrm{ME}$ as the reducing agent throughout the SeMet protein purification and crystallization.

The native x-ray diffraction dataset $\left(d_{\min }=2.6 \AA\right)$ was collected at the Macromolecular Diffraction at the Cornell High Energy Synchrotron Source (MacCHESS) A1 beamline, and a multiwavelength anomalous dispersion dataset $\left(d_{\min }=3.0 \AA\right)$ was obtained at the National Synchrotron Light Source X-4 station at $100 \mathrm{~K}$. Reflection data were integrated and scaled with the HKL2000 program (34). Real-space locations of all twelve of the expected selenomethionines in the asymmetric unit were placed using SOLVE (35). The initial model was built, using Quanta 2000 (Accelrys, San Diego, CA), into a density-modified map generated using the DM module of the Collaborative Computational Project 4 suite $(36,37)$. This model was subjected to rounds of simulated annealing, energy minimization, individual B-factor refinement in crystallography NMR software (version 1.1) against the native dataset interspersed with manual rebuilding to produce a model with final values of $R_{\text {work }}=22.7 \%$ and $R_{\text {free }}=26.7 \%$. Noncrystallographic symmetry was not employed during the refinement. Electron density was weak or absent for a significant number of side chains: protomers A and B had 2102 and 2091 atoms built with 32 and 46 side-chains partially disordered (110 and 175 atoms not modeled), respectively. The figures were created with Ribbons 3.22 and GRASP1.2 software (the GRASP surface was created with a model in which the disordered parts of side chains with $\beta$-carbon density were modeled to most appropriately reflect surface charge distributions) $(38,39)$. PROCHECK and SFCHECK were used to inform model building and validate the final model $(40,41)$.

Topo IB Relaxation Assays-Negatively supercoiled pBR322 was prepared using a GigaKit (Qiagen) $(14,42)$. The pBR322-resuspended pellet was dialyzed against $20 \mathrm{~mm}$ Tris, $\mathrm{pH} 8.0,1 \mathrm{~mm}$ EDTA and concentrated on a $30 \mathrm{~K}$ polyethersulfone membrane (Vivaspin) to 0.85 $\mu \mathrm{g} / \mathrm{ml}$. To obtain undamaged pBR322, it is essential to never freeze the DNA. The reaction buffer included $35 \mathrm{~mm}$ Tris, $\mathrm{pH} 7.4,50 \mathrm{~mm} \mathrm{NaCl}$, $1.85 \mathrm{~mm} \mathrm{MgCl}_{2}, 5 \mathrm{~mm}$ spermidine, $100 \mu \mathrm{g} / \mathrm{ml}$ bovine serum albumin, 9 $\mu \mathrm{g} / \mathrm{ml}$ tRNA, $6.5 \%$ glycerol, $5.5 \mathrm{~mm} \beta \mathrm{ME}, 1 \mathrm{~mm}$ ATP, 2 mM HEPES, 0.1 mM EDTA, $300 \mathrm{~nm}$ pBR322, and 0.6-6.0 $\mu \mathrm{M}$ GyrA-CTD (E. coli (532841 ) or $B$. burgdorferi; the results are identical with or without $\mathrm{His}_{6}$ tags). T4 DNA ligase (100 units, New England Biolabs) was added to each reaction. After $10 \mathrm{~min}$, DNA Topo IB from vaccinia virus (10 units, Epicentre) was added to a final volume of $10 \mu \mathrm{l}$, and the reactions were incubated for $4-6 \mathrm{~h}$ at $37^{\circ} \mathrm{C}$. Phenol:chloroform:isoamyl alcohol $(25$ : 24:1) buffered to $\mathrm{pH} 8.0$ was used to extract the protein from the reactions, followed by ethanol precipitation. The DNA was resuspended in $20 \mathrm{~mm}$ Tris, $\mathrm{pH} 8.0,1 \mathrm{~mm}$ EDTA. Gels with $\sim 0.5 \mu \mathrm{g}$ of DNA/lane (1.1\% agarose in $1 \times$ TPE (Tris phosphate, EDTA) or $1 \times$ TAE (Tris acetate, EDTA)) were run at $4 \mathrm{~V} / \mathrm{cm}$ for $10 \mathrm{~min}$ and then $1.0 \mathrm{~V} / \mathrm{cm}$ for 19-21 h. After ethidium bromide staining, the topoisomer bands were quantitated in ImageQuant TL (Amersham Biosciences) and plotted in Kaleidagraph (Synergy Software), where they were fit to gaussian curves.

\section{RESULTS}

Structure Analysis-Crystals of the E. coli GyrA-CTD (residues 531-853 plus an N-terminal hexahistidine tag) produced diffraction data to a limiting resolution of $2.6 \AA$ (supplemental Table I). Experimental phases were determined by multiwavelength anomalous dispersion phasing using selenomethioninelabeled protein crystals and then applied to a native data set. The final model includes two protomers/asymmetric unit, each comprising residues 535-841, with a large disordered loop from 555 to 576 in protomer A and 563 to 575 in protomer B (Fig. 1C). This disordered loop, hereafter referred to as the $\beta$-CA loop, contains a known trypsin cleavage site plus the "GyrA box," an indel used to distinguish GyrA from ParC replete with positively charged residues $(43,44)$. The version of the GyrA-CTD that yielded the highest quality diffraction data contained an R562C mutation in the $\beta$-CA loop, but the loop was similarly disordered in the $2.9-\AA$ structure of the nonmutated protein (data not shown); because the two proteins were indistinguishable structurally, we used the R562C protein for complete refinement and structural interpretation. We note that the dissociation constant of the GyrA-CTD for DNA is unaffected by the presence of the R562C mutation (data not 
A

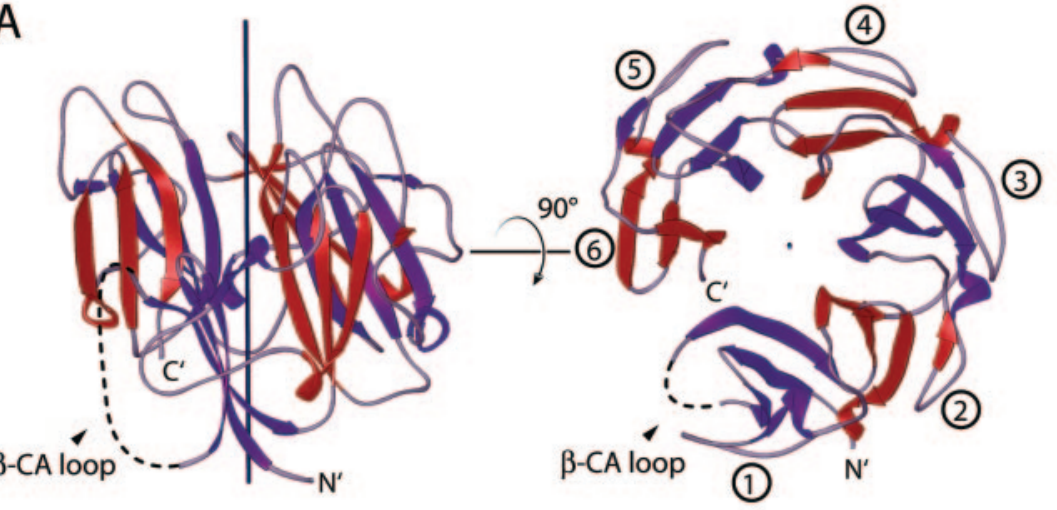

B

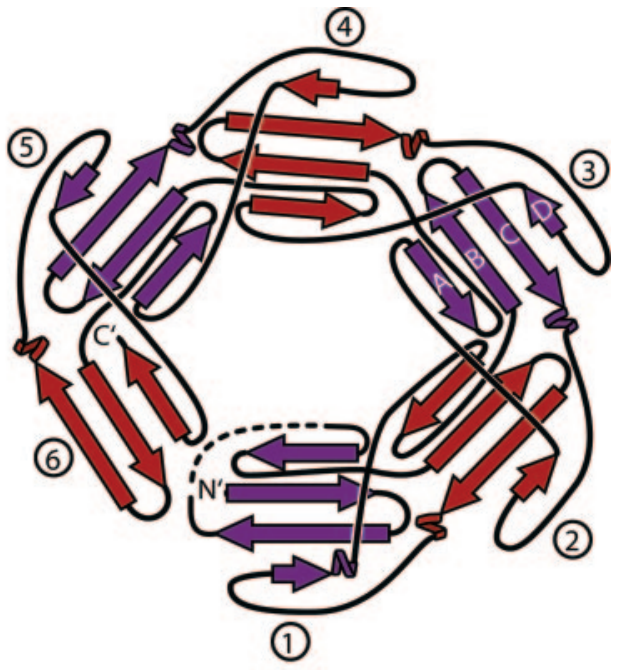

C

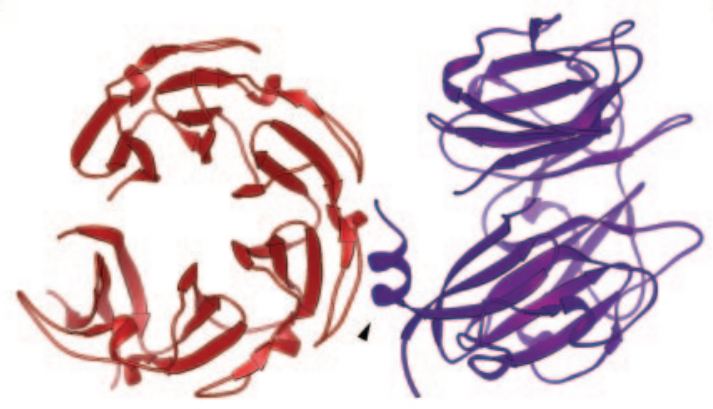

D

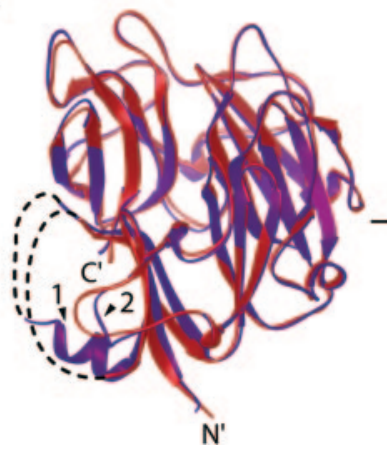

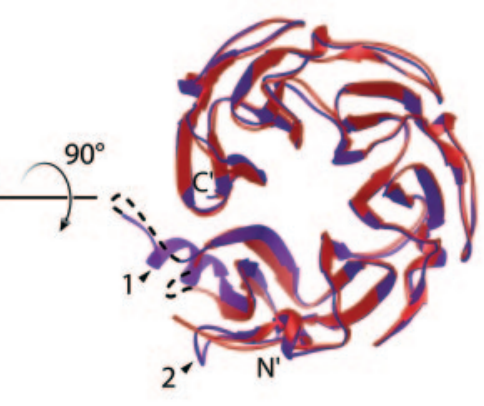

FIG. 1. Structure analysis of $\boldsymbol{E}$. coli GyrA C-terminal domain. $A$, spiraling $\beta$-pinwheel structure of the $E$. coli GyrA-CTD residues $535-841$, with the disordered $\beta$-CA loop (555-576) represented by a dotted line. The blade subdomains (four-stranded antiparallel $\beta$-sheet and a $3_{10}$ helix) are labeled 1-6 and alternate in color from red to blue. The choice of blade nomenclature used here for the spiraling $\beta$-pinwheel preserves the four-stranded sheet with strands labeled from the center proximal outward following the convention for $\beta$-propellers. Note that the topology for all three CTD structures discussed in this paper is identical, despite the different blade and strand nomenclature used by Corbett et al. (28, 32). $B$, topology diagram illustrating interdigitation of blades. $C$, asymmetric unit of the $E$. coli GyrA-CTD structure. Monomer A is in red, and monomer $\mathrm{B}$ is in blue. The perhaps spurious packing $\alpha$-helix formed from the $\beta$-CA loop is indicated by the wedge. $D$, C $\alpha$ superposition of monomers A and B. The r.m.s.d. is $1.2 \AA$ and falls to $0.7 \AA$, when the $\alpha$-helix (wedge 1) in B and the loop that abuts it (wedge 2) are excluded from the calculation. Dashed lines indicate disordered loops spanning residues 555-576 and 563-575 in protomers A and B, respectively.

shown). The two protomers possess nearly identical overall structures and can be superimposed with a $\mathrm{C} \alpha$ r.m.s.d. of $1.2 \AA$. Significant structural differences between the two are localized entirely to flexible elements located at the crystal packing interface, namely the ordering of a small stretch of the $\beta$-CA loop (residues 555-562) in protomer B into a short $\alpha$-helix and the minor adjustment of a nearby loop (residues 608-617) (Fig. $1 D$, wedges). Upon removal of these parts from the superposition, the r.m.s.d. drops to $0.7 \AA$. Because the short helix in protomer B appears to be a crystal packing artifact, we have used protomer A for all figures, except Fig. 1, $C$ and $D$. The crystal structure of the $E$. coli GyrA-NTD (29) was ordered through residue 522, thus only 12 amino acids are missing between the structures of the two domains of GyrA (Fig. 2A).

The overall fold of the $E$. coli GyrA-CTD is similar to that recently described for the structure of the B. burgdorferi GyrA-CTD (32), with both bearing a superficial similarity to the well known $\beta$-propeller (45). Similar to $\beta$-propeller proteins, the GyrA-CTDs contain serially repeated subdomains known as "blades," each of which is composed of a fourstranded antiparallel $\beta$-sheet (Fig. 1, $A$ and $B$ ). Closer inspection, however, reveals that the topology of the blades in the GyrA-CTDs is completely different from that in $\beta$-propeller proteins; this distinction prompted Corbett et al. (32) to describe the GyrA-CTD fold as a $\beta$-pinwheel. Whereas the $\beta$-strands that make up the blades in a $\beta$-propeller reside on a contiguous stretch of polypeptide sequence, the $\beta$-pinwheel is a more interdigitated structure, with the polypeptide chain
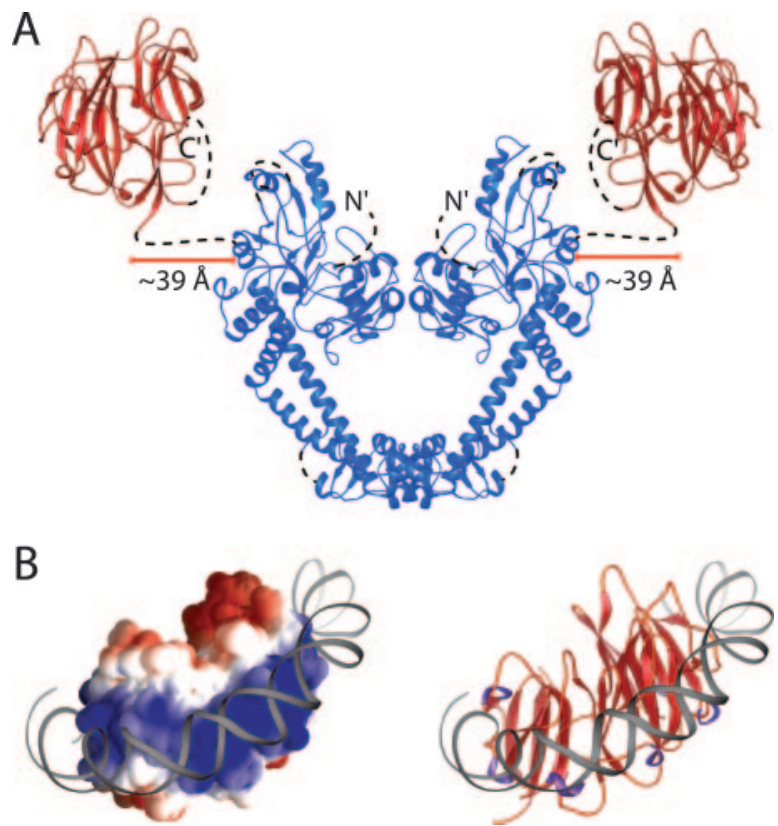

FIG. 2. Models. A, the E. coli GyrA-NTD (ordered from 30-522) (29) and the E. coli GyrA-CTD (ordered from 535-841) showing the largest possible distance of $39 \AA$ separating the two domains. $B$, model suggesting how DNA might wrap about the $E$. coli GyrA-CTD to impart right-handed superhelicity on the DNA. Left, DNA modeled onto the electrostatic potential surface. 

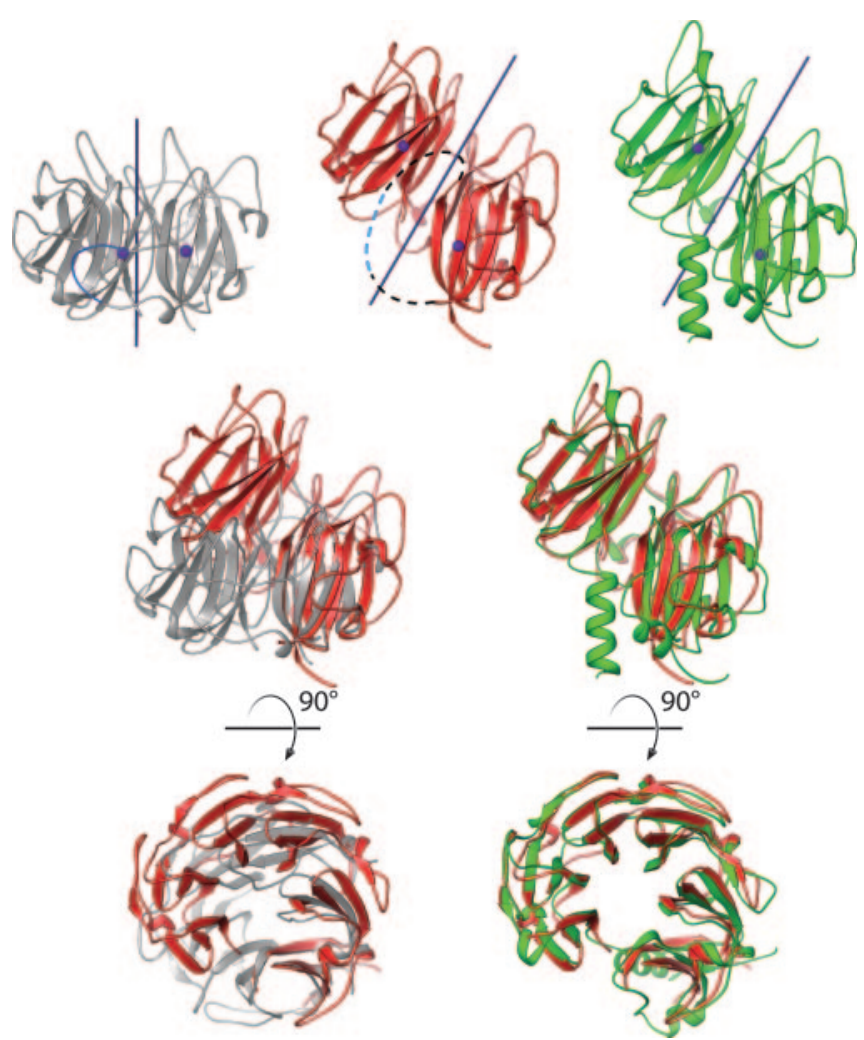

FIG. 3. Superpositions of C-terminal domains. $A, \mathrm{C} \alpha$ superpositions using blade 1 of the $E$. coli GyrA-CTD (red), the B. burgdorferi GyrA-CTD (gray) (32), and the B. stearothermophilus ParC-CTD (28) (green), depicting the difference in overall orientation of the blades in the two gyrase structures and the striking similarity of the $E$. coli GyrA-CTD and the B. stearothermophilus ParC-CTD. The screw axis (dark blue cylinder) was determined by serial superposition of blades normalized to the origin and averaging the resulting rotation axis vectors. Blue spheres represent the center of masses of blades 1 and 6 ; the distance along the screw axis separating these centers of mass is 11 $\AA$ for the $E$. coli GyrA-CTD. The GyrA box (cyan) is a portion of the $\beta$-CA loop, ordered in the B. burgdorferi GyrA-CTD, disordered in the case of the $E$. coli GyrA-CTD, and by definition absent in the ParC-CTD.

meandering back and forth between adjacent blades (Fig. 1, $A$ and $B$ ).

Although each blade in the $\beta$-pinwheel fold of the $E$. coli GyrA-CTD is nearly identical to its counterpart in the $B$. burgdorferi GyrA-CTD $\beta$-pinwheel (average r.m.s.d. $=1.5 \AA$ ), the spatial arrangement differs significantly; while the blades of the $B$. burgdorferi GyrA-CTD form a toroidal $\beta$-pinwheel, the blades of the $E$. coli GyrA-CTD are arranged in an ascending spiral around a screw axis (Fig. 3). If the B. burgdorferi GyrACTD has the appearance of a flat washer, then the $E$. coli GyrA-CTD has the appearance of a lock washer. Consequently, a global superposition of the B. burgdorferi and $E$. coli proteins yields an r.m.s.d. of $4.59 \AA$ (5.45 $\AA$ for protomer B), in marked contrast to the excellent correspondence of blade-to-blade superpositions. The significant divergence in overall shape can clearly be observed in a $\mathrm{C} \alpha$ superposition using the $\mathrm{N}$-terminal blade of each structure (Fig. 3). To gain a more quantitative estimate of the difference in helicity of the two proteins, we computed a screw axis (Fig. 3, blue rod) and measured the displacement of the center of mass for blades 1 and 6 (Fig. 3, spheres) along the axis. Although the $B$. burgdorferi $\beta$-pinwheel has almost no axial displacement $(0.04 \AA)$ from blade 1 to 6 , the $E$. coli $\beta$-pinwheel exhibits pronounced axial displacement $(11.03 \AA)$. Consonant with the axial displacement of the propeller blades, the interface between blades 1 and 6 is much more extensive in the $B$. burgdorferi GyrA-CTD structure than in the $E$. coli structure $\left(2,150 \AA^{2}\right.$ of solvent-inaccessible surface
A

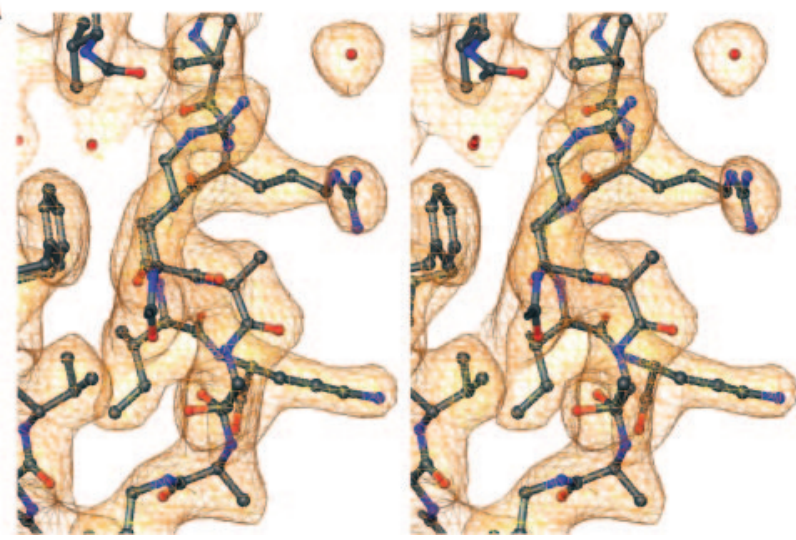

FIG. 4. A stereo view of a small region of the final model and corresponding electron density map. The loop in blade 4 spanning the $3_{10}$-helix to the $\mathrm{D}$ sheet is displayed, and its $\sigma_{A}$-weighted $2 F_{o}-F_{c}$ map is contoured at $1 \sigma$.

versus $466 \AA^{2}$, respectively). In the $B$. burgdorferi structure, a significant fraction of this interface is contributed by the GyrA box on the $\beta$-CA loop $\left(991 \AA^{2}\right.$ ), whereas the whole loop is disordered in the $E$. coli GyrA-CTD.

The Bacillus stearothermophilus ParC-CTD (Topo IV) structure, reported while this work was under review, also has a $\beta$-pinwheel fold with the blades arranged in an ascending spiral (28); the E. coli GyrA-CTD and the B. stearothermophilus ParCCTD superimposed with an overall r.m.s.d. of $1.5 \AA$ (Fig. 3). The most notable structural difference between these two domains is the presence of $\alpha$-helices in the $\beta$-CA loop and at the $\mathrm{C}$ terminus of the ParC-CTD. The latter packs against strand A of blade 1, resulting in a much more extensive interaction between blades 1 and $6\left(1086 \AA^{2}\right)$ than in the $E$. coli GyrA-CTD structure $\left(466 \AA^{2}\right)$.

It is formally possible that the differences in overall fold between these three structures result from crystal packing. Two independent lines of evidence suggest this is not likely. Protomers A and B of the E. coli GyrA-CTD asymmetric unit are virtually identical in fold, despite packing differently within the unit cell (Fig. 1, $C$ and $D$, and supplemental Fig. 3). Further, the B. stearothermophilus ParC-CTD, which also shares this spiral fold, packs in yet another form (space group $P 3_{2}$ ) (supplemental Fig. 3) (28).

To gain insight into the charge density and distribution on the surface of the $E$. coli GyrA-CTD, we calculated its surface electrostatic potential (Fig. 2B). Strikingly, a positively charged strip extends along the midriff of the protein, following the right-handed superhelical rise of the $\beta$-pinwheel. When DNA is modeled into the structure so as to maximize its electrostatic interactions with this positive strip, the right-handed superhelical rise in the positive strip imparts a corresponding superhelical rise in the bound DNA (Fig. $2 B$ ). Interestingly, although the $B$. burgdorferi structure also contains a positive strip (32), it lacks a spiral sense and hence is not expected to impart any superhelical rise upon its bound DNA. In either case, most of the predicted DNA contacts can be attributed to the preponderance of arginines and lysines on the long loops connecting the $3_{10}$ helix of one propeller blade with the outermost $\beta$-strand in its neighbor (Figs. $1 A$ and 4 ). It is conceivable and perhaps even likely that these basic loops undergo some local conformational adjustment upon DNA binding but unlikely that they change so drastically as to alter the overall course of the positive strip. The right-handed helical arrangement of blades from $\mathrm{N}$ to $\mathrm{C}$ terminus suggests a model for DNA wrapping, whereby binding along the midriff of the protein surface imparts right-handed superhelicity in the DNA (Fig. 2B). 


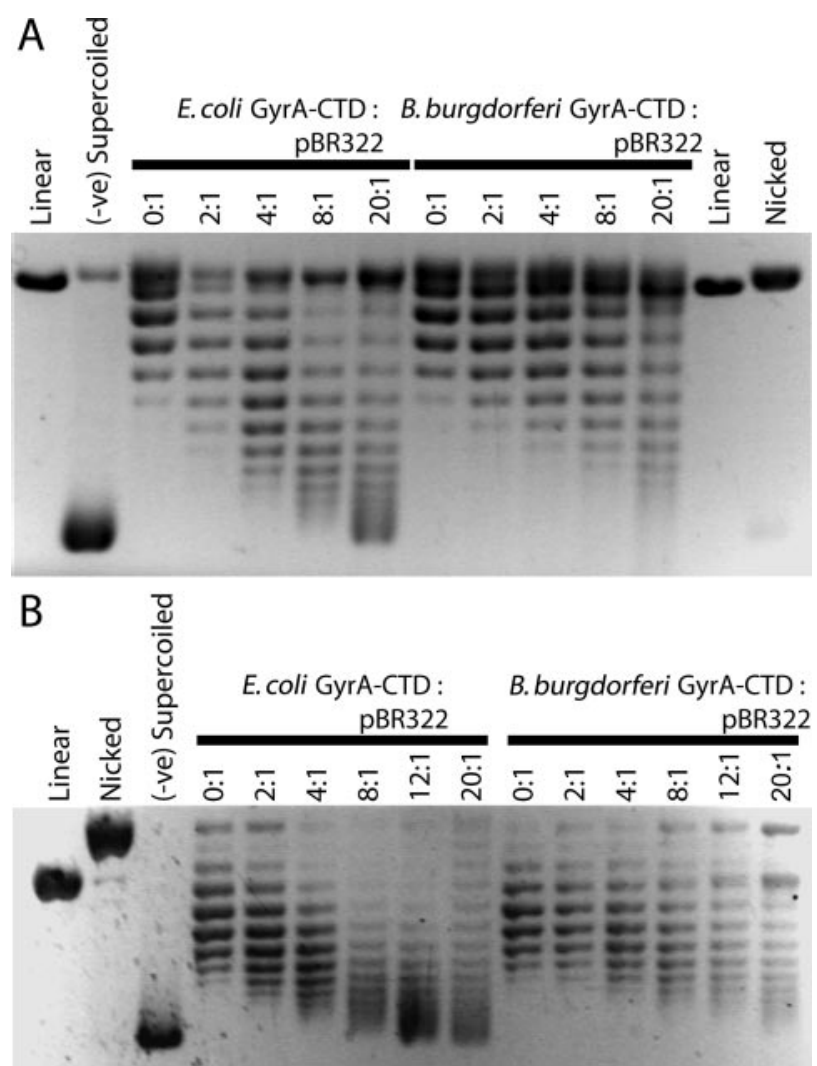

FIG. 5. Topoisomerase I relaxation assay. Vaccinia virus Topo IB was used to relax pBR322 (300 nM) in the presence of either the $E$. coli or B. burgdorferi GyrA-CTD at increasing molar ratios of protein, trapping the local superhelical state induced by protein binding. Nicked and linearized controls were obtained by digestion of pBR322 with N.BpuI or HindIII, respectively. A, samples run on a $1.1 \%$ agarose gel $(1 \times$ TPE buffer) and stained with ethidium bromide. $B$, samples run on a $1.1 \%$ agarose, $0.6 \mu \mathrm{g} / \mathrm{ml}$ chloroquine gel ( $1 \times \mathrm{TAE}$ buffer) and stained with ethidium bromide. The sign of the linking number changes were determined to be positive from the increase in mobility of the topoisomers in the chloroquine gel $(B)$ compared with the non-chloroquine gel (A). Further increases in mobility were observed on a $3 \mu \mathrm{g} / \mathrm{ml}$ chloroquine gel (supplemental Fig. 1A). The bands on both gels were quantified and fit to gaussian curves to determine the $\Delta \mathrm{Lk} /$ protein (supplemental Fig. $1 B$ ).

Topo I Readout of Wrapping-To compare changes in DNA topology upon binding of the $E$. coli or the B. burgdorferi GyrA-CTDs, Topoisomerase IB was used to relax pBR322 plasmids in the presence of either CTD (Fig. 5) (14, 42). Compensatory torsional force introduced by local deformation of DNA topology by a given protein binding event is relaxed by the Topo IB. When the proteins are extracted from the DNA, the sum of local deformations is observable as a global topological change. A similar experiment (plasmid nicking-religation) yielded the first evidence that the E. coli GyrA-CTD (residues 572-875) generates $(+)$ superhelical wrapping of DNA, resulting in a $\Delta \mathrm{Lk}$ of $+0.025 /$ protein (15). However, in light of the present structural data, the precise interpretation of this value is confounded by the fact that the protein used in these experiments was missing a sizeable portion of blade 1 (15).

Dissociation constants for both the $E$. coli $(1.17 \pm 0.09 \mathrm{~nm})$ and the B. burgdorferi ( $12 \pm 1 \mathrm{nM})$ GyrA-CTDs were measured by electrophoretic mobility shift analysis (data not shown) to ensure the Topo I assay was performed in the stoichiometric binding regime for both proteins. In our experiments, the two GyrA-CTD proteins showed significant differences in their wrapping behavior. With Topo I, the $E$. coli GyrA-CTD introduced $(+)$ supercoils, which can be interpreted as the protein wrapping DNA into right-handed $((+)$ solenoidal $)$ superhelices, with a $\Delta$ Lk of $+0.8 \pm 0.1$ protein (Fig. $5 A$ and supplemental Fig. 1). A more modest $\Delta \mathrm{Lk}$ of $+0.3 \pm 0.1$ /protein is observed for the B. burgdorferi GyrA-CTD with Topo I (Fig. $5 B$ and supplemental Fig. 1). The sign of the linking number changes was established by running chloroquine gels (Fig. $5 B$ and supplemental Fig. 1).

\section{DISCUSSION}

Recent structural findings on CTDs from B. burgdorferi DNA gyrase and $B$. stearothermophilus Topo IV revealed $\beta$-pinwheel folds that can adopt two markedly distinct overall conformations, a planar one bearing resemblance to a flat washer and a spiral one resembling a lock washer $(28,32)$. That topoisomerases from different functional classes should display such a clear structural distinction led quite naturally to the suggestion that gyrase CTDs are flat and Topo IV CTDs are spiral, and that this difference gives rise to the distinctive classspecific functional characteristics of each (28). The present structural findings dispel the notion that the structure/function relationships in topoisomerase CTDs follow such a simple pattern. Specifically, here we report that the CTD from $E$. coli DNA gyrase has a spiral (not flat) arrangement of blades. Thus, the $E$. coli GyrA-CTD is more closely related in overall structure to the paralogous Topo IV CTD than to the orthologous B. burgdorferi GyrA-CTD.

The present structure furthermore dispels the notion that the GyrA box, which is present in GyrA-CTDs (refer to Fig. 1) but lacking in Topo IV-CTDs, is responsible for stabilizing the flat structure by lashing blades 1 and 6 together (28). It is possible that the particular GyrA box sequence in the $B$. burgdorferi GyrA-CTD does stabilize the flat structure, but this cannot be the role of the GyrA box in general, because the E. coli GyrA-CTD contains a GyrA box and yet adopts a spiral structure. It is worthy of note that the GyrA box of the $E$. coli protein (QRRGGKG) is a perfect match to the consensus sequence ( $\mathrm{Q} X X \mathrm{GGXG}$, where $X$ is a positively charged amino acid) (44); whereas the GyrA box in B. burgdorferi (QGTGGKG) diverges further from the consensus than 85 of 87 GyrA orthologs (Supplementary Fig. 2). Also noteworthy is the fact that the $\beta$-CA loop, including the GyrA box, is disordered in the $E$. coli GyrA-CTD, which raises the possibility that this entire segment of the CTD becomes ordered upon docking with the remainder of gyrase or upon binding DNA.

Having established that the flat versus spiral structural divergence transcends class distinctions among topoisomerases, the question remains as to whether the two divergent shapes give rise to distinct and definable functional roles. Below, we will focus first on the implications for gyrase function and will then return to the issue of Topo IV mechanism. A predicted $\beta$-pinwheel CTD is found in all known Topo II enzymes capable of introducing (-) supercoils (9). Removal of the GyrA-CTD from $E$. coli gyrase results in loss of the bias toward introduction of (-) supercoils but retention of core Topo II activity (7). Finally, a subfragment of the $E$. coli CTD (residues 572-875) is able to complement a CTD-less gyrase variant (residues $6-571$ ) (12), suggesting the CTD behaves as a plug-in structural module of gyrase, rather than an inextricably linked structural component. Taken together, these results lend strong support to the notion that the GyrA-CTD is responsible for the (-) supercoiling bias of DNA gyrases and bolster confidence in the extrapolation of results from the isolated CTDs to full-length gyrase.

Gyrase CTDs, both flat and spiral, possess a stripe of positive charge about their midriff that is the only obvious locus for interaction with a contiguous stretch of duplex DNA. Consistent with this notion, fluorescence resonance energy transfer experiments on the B. burgdorferi GyrA-CTD indicate that the 

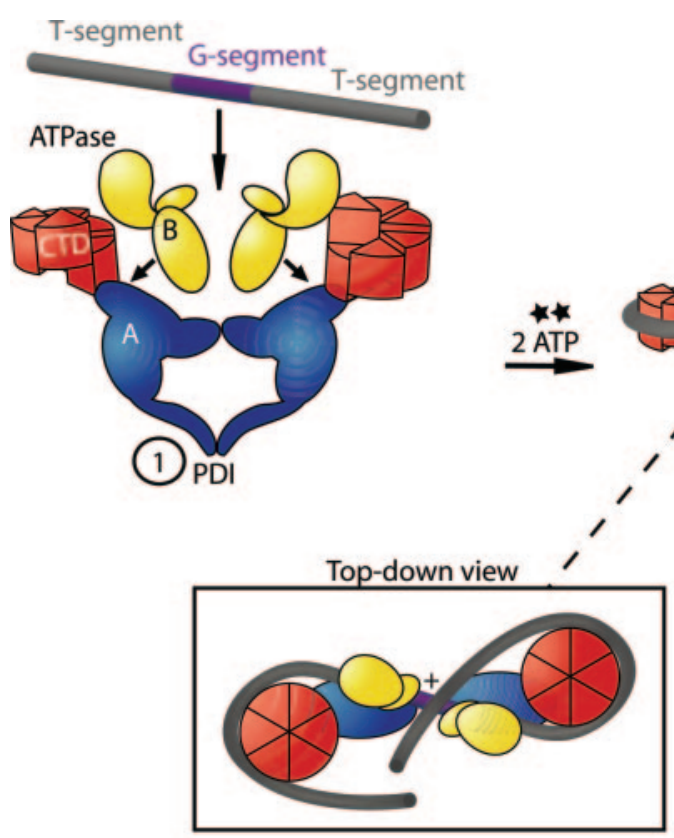

(2)
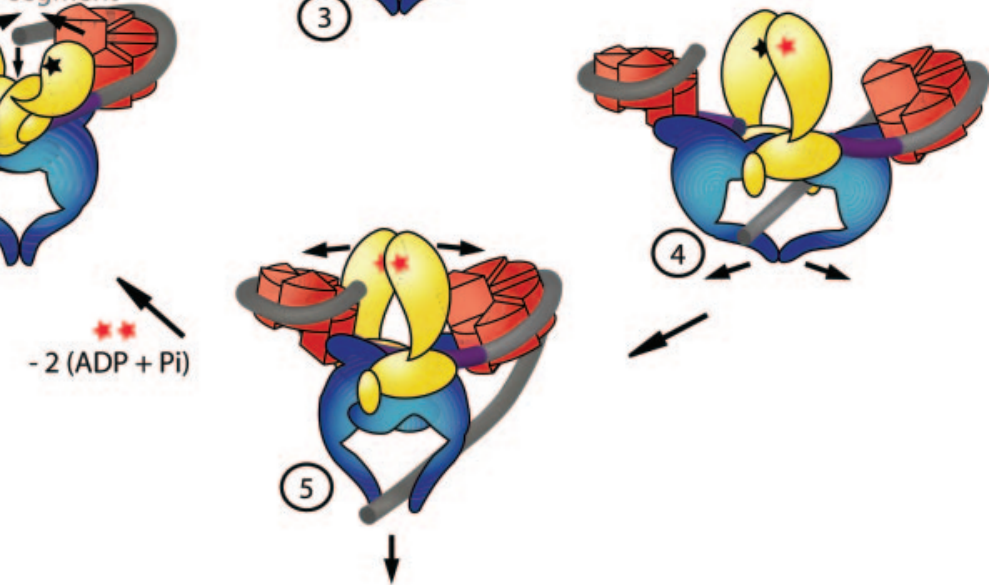

FIG. 6. Model of the mechanism by which the GyrA-CTD imparts a bias for relaxing $(+)$ supercoils and introducing (-) supercoils. 1 , the DNA binds to gyrase $\left(\mathrm{A}_{2} \mathrm{~B}_{2}\right)$ cooperatively, with the cleavage/reunion core binding to the G-segment. GyrA is blue and red, for the $\mathrm{N}$ and C-terminal domains, respectively, and GyrB is yellow. 2, the GyrA-CTDs present segments of G-segment flanking DNA to the cleavage/reunion core as potential T-segments (inset). Top-down view of T-segment presentation. 3, only one T-segment is captured by ATP (black star) binding-mediated GyrB dimerization and passed through the transient cleaved G-segment gate into the central cavity of the GyrA-NTD. 4, the selected T-segment is passed through the primary dimerization interface $(P D I)$ and release of $\mathrm{ADP}$ (red star) and $\mathrm{P}_{\mathrm{i}}$ resets the system. In general, type II topoisomerase activity changes the linking number in $\Delta \mathrm{Lk} \pm 2$ steps via sign interconversion at the node. By enforcing the $(+)$ node as a DNA substrate requirement for gyrase, the GyrA-CTD is responsible for (+) supercoil relaxation and (-) supercoil induction.

domain has the ability to induce bends in DNA (32). Similar fluorescence resonance energy transfer and single-molecule DNA-contraction experiments on the $E$. coli GyrA-CTD reach the same conclusion (data not shown). Although the B. burgdorferi and the $E$. coli GyrA-CTDs both wrap DNA, the manner of wrapping is not identical, because the B. burgdorferi CTD does not alter the average linking number of plasmid DNA in relaxation experiments described above to the same extent as the $E$. coli CTD. Two different modes of CTD-mediated conformational changes in the bound duplex DNA could account for these changes in linking number, overtwisting, or $(+)$ writhe induction via wrapping of the DNA around the CTD, or some combination of both. The relatively modest $\Delta \mathrm{Lk} /$ protein of +0.3 for the B. burgdorferi GyrA-CTD could result entirely from the twist imparted by a protein binding DNA about its radial surface. On the other hand, overtwisting alone is highly unlikely to account for the observed $\Delta \mathrm{Lk} /$ protein imparted by the E. coli CTD, because its large magnitude $(+0.8 / E$. coli GyrACTD) would require the introduction of nearly one complete additional turn over the $<50$ base-pairs of DNA (4-5 turns) estimated to be bound to the CTD $(18,21)$, as compared with relaxed, B-form DNA. If overtwisting alone cannot account for the observed linking number change, then writhe, most likely in the form of $(+)$ solenoidal superhelical wrapping, must play a role. Such wrapping of DNA around a roughly cylindrical surface is well precedented in the nucleosome core particle (46). The nucleosomal DNA is wrapped in a left-handed $((-)$ solenoidal) superhelix (-1.67 superhelical turns) in opposition to slight overtwisting (10.17 bp/turn versus $10.5 \pm 0.1$ for canonical B-DNA, for a total $\Delta \mathrm{Lk} /$ nucleosome of -1.2 ) (46). We note that, by adjusting the magnitudes of writhe and twist from the nucleosome to the DNA size regime of that bound by the $E$. coli GyrA-CTD and assuming the sign of twist and writhe changes are both $(+)$, one arrives at a $\Delta \mathrm{Lk} /$ protein of +0.8 (twist $=+0.2$, and writhe $=+0.6$ ). Although actual magnitudes of writhe and twist cannot be determined from our pres-

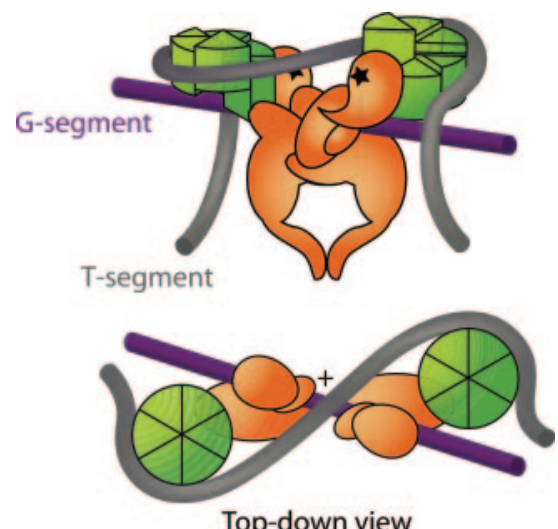

FIG. 7. Model by which the ParC-CTD (green) of Topo IV (ParC/ ParE) (orange) imparts a bias for $(+)$ node processing. There is no preference for the superhelical state of the G-segment (26), whereas (+) solenoidal superhelical T-segments are preferentially captured and processed through the G-segment by the right-handed spiraling DNA binding surface of the ParC-CTD.

ent data, we favor a model with a preponderance of the $E$. coli GyrA-CTD +0.8 $\Delta \mathrm{Lk} /$ protein superhelicity in the form of writhe, because the kind of gentle superhelical writhe suggested by the structure (Fig. $2 B$ ) is less energetically costly than drastic overwinding. Importantly, the spiral sense in the $E$. coli GyrA-CTD is (+), consistent with the observed (+) linking number change in the Topo I assay. Full-length $E$. coli gyrase, which has two GyrA-CTDs, also introduces a (+) linking number change in DNA in a similar assay $(14,15)$. The change in linking number is +0.7 to 0.8 per enzyme, indicating the extent of DNA wrapping by each CTD must be diminished or only one CTD of the two in gyrase is engaged with DNA at a time. Thus, in summary, we propose that the spiral positively charged stripe about the midriff surface of the E. coli GyrACTD is predisposed toward binding DNA containing comple- 
mentary superhelicity, whereas the flat B. burgdorferi GyrACTD simply prefers to bind DNA in a more flat, co-planar fashion

The fact that the $B$. burgdorferi GyrA-CTD alone imparts significantly less supercoiling bias raises the possibility that this gyrase might function in a subtly different manner from other gyrases. $B$. burgdorferi is unique among all known DNA gyrases in that its CTD is expressed as an independent gene product, which incidentally was initially discovered in a genetic screen for proteins that bind the telomeres of the linear plasmid of $B$. burgdorferi (47). It is not known whether this unique structural feature results in a functional role distinct from other gyrases, because the supercoiling activity of the isolated fulllength enzyme has not been reported. The B. burgdorferi GyrACTD can complement HU protein loss in $E$. coli, suggesting that the CTD might be involved in genome compaction or another of the many physiological functions of $\mathrm{HU}$ protein $(47,48)$. To gain some insight into the evolutionary context of $B$. burgdorferi GyrA, we constructed a consensus of the most parsimonious phylogenetic trees with all GyrA and ParC genes from completed genomes (supplemental Fig. 2) (49). Full GyrA from B. burgdorferi resides in a monophyletic group with the other species of spirochetes; however, the B. burgdorferi GyrA-CTD is no longer monophyletic with the GyrA-CTD of other spirochetes, and moreover, it is an outlier from most gyrase A proteins. Further biochemical studies on the $B$. burgdorferi gyrase will be required to resolve the precise role of its planar GyrA-CTD.

We propose a model wherein the wrapping is largely or wholly performed by the C-terminal domain of $E$. coli gyrase A (Fig. 6). In this model, the cleavage/reunion core binds $\sim 35$ base pairs as the G-segment and each GyrA-CTD wraps $\sim 45$ base pairs of contiguous DNA, positioning two possible Tsegments for capture $(14,21,50)$. Further, we hypothesize that the highly positively charged GyrA box directs DNA from the G-segment binding cleft to the GyrA-CTD wrapping surface, forcing presentation of a contiguous T-segment. Inspection of the $E$. coli GyrA-CTD structure implies righthanded superhelicity is imparted on the DNA as it binds about the radial surface of the GyrA-CTD, following the helical path of the blades. In particular, the GyrA-CTD binds the DNA with right-handed $((+)$ solenoidal) superhelicity that enforces presentation of a $(+)$ node to the cleavage/reunion core, leading to the introduction of $(-)$ supercoils after Tsegment passage via nodal sign inversion (50). This model further differs from that proposed by Corbett et al. (32) in that it does not require positioning of the GyrA-CTD by packing against the rest of gyrase to impose directionality of the wrap, although it does not preclude such packing.

Unexpectedly, the $E$. coli GyrA-CTD shares its distinctive spiral structure with the evolutionarily (supplemental Fig. 2) and functionally distant CTD from B. stearothermophilus ParC, yet diverges in this structural feature from the presumably more closely related B. burgdorferi GyrA-CTD. Can some common role for the spiral structure be envisaged in the E. coli GyrA-CTD and B. stearothermophilus ParC, despite the overall functional differences between these two classes of type II topoisomerases? In both, $(+)$ toroidal superhelical wrapping of the T-segment by the spiral CTDs could underlie the topologically biased activity of the enzymes: $(-)$ supercoil induction by gyrase and preference for processing $(+)$ nodal crossovers during decatenation and supercoil relaxation by Topo IV (Fig. 7). The essential mechanistic difference between gyrase and Topo IV stems from the contiguity of the G-segment to the T-segment presented; while gyrase enforces this contiguity, Topo IV does not (25). In light of the present structural results, it is tempting to speculate further that the
GyrA box might play a role in the enforcement of $\mathrm{G}$ and $\mathrm{T}$ segment contiguity by gyrase. This highly charged stretch may electrostatically guide the G-segment from the cleavage/ reunion core binding cleft to the CTD in the case of gyrase, whereas in Topo IV, the absence of this concentrated charge in the $\beta$-CA loop may destabilize or prevent such presentation of a continuous stretch of DNA. Unlike gyrase, Topo IV appears to bind a T-segment only transiently during processing (51). The ParC-CTD is believed to be responsible for T-segment selection (28), and the $E$. coli ParC-CTD is capable of inducing bends in DNA (32). Taking these earlier findings into account, we add that the right-handed spiraling nature of the ParC-CTD could be directly responsible for selection of T-segments that form (+) nodes (Fig. 7). Our model explains that the selectivity for $(+)$ supercoiled DNA by Topo IV occurs during processing, despite the absence of any such selectivity in initial G-segment binding (26).

Acknowledgments-We thank Bill Miller, Randy Abramowitz, and colleagues at the CHESS A1 beamline and the National Synchrotron Light Source X4A beamline for expert assistance and J. Blumenstiel for help constructing the phylogenetic trees. This work is based upon research conducted at the CHESS, which is supported by the National Science Foundation under award DMR 0225180, using the MacCHESS facility, which is supported by award RR-01646 from the National Institutes of Health through its National Center for Research Resources. Use of the National Synchrotron Light Source, Brookhaven National Laboratory, was supported by the United States Department of Energy, Office of Science, Office of Basic Energy Sciences, under Contract No. DE-AC02-98CH10886.

\section{REFERENCES}

1. Wang, J. C. (1996) Annu. Rev. Biochem. 65, 635-692

2. Champoux, J. J. (2001) Annu. Rev. Biochem. 70, 369-413

3. Espeli, O., and Marians, K. J. (2004) Mol. Microbiol. 52, 925-931

4. Zechiedrich, E. L., Khodursky, A. B., Bachellier, S., Schneider, R., Chen, D., Lilley, D. M. J., and Cozzarelli, N. R. (2000) J. Biol. Chem. 275, 8103-8113

5. Noble, C. G., and Maxwell, A. (2002) J. Mol. Biol. 318, 361-371

6. Hockings, S. C., and Maxwell, A. (2002) J. Mol. Biol. 318, 351-359

7. Kampranis, S. C., and Maxwell, A. (1996) Proc. Natl. Acad. Sci. U. S. A. 93, 14416-14421

8. Caron, P. R., Watt, P., and Wang, J. C. (1994) Mol. Cell. Biol. 14, 3197-3207

9. Caron, P. R., and Wang, J. C. (1994) Adv. Pharmacol. 29B, 271-297

10. Austin, C. A., and Marsh, K. L. (1998) BioEssays 20, 215-226

11. Reece, R. J., and Maxwell, A. (1991) J. Biol. Chem. 266, 3540-3546

12. Critchlow, S. E., and Maxwell, A. (1996) Biochemistry 35, 7387-7393

13. Liu, L. F., and Wang, J. C. (1978) Proc. Natl. Acad. Sci. U. S. A. 75, 2098-2102

14. Kampranis, S. C., Bates, A. D., and Maxwell, A. (1999) Proc. Natl. Acad. Sci. U. S. A. 96, $8414-8419$

15. Reece, R. J., and Maxwell, A. (1991) Nucleic Acids Res. 19, 1399-1405

16. Bates, A. D., O’Dea, M. H., and Gellert, M. (1996) Biochemistry 35, 1408-1416

17. Rau, D. C., Gellert, M., Thoma, F., and Maxwell, A. (1987) J. Mol. Biol. 193, $555-569$

18. Heddle, J. G., Mitelheiser, S., Maxwell, A., and Thomson, N. H. (2004) J. Mol. Biol. 337, 597-610

19. Kirkegaard, K., and Wang, J. C. (1981) Cell 23, 721-729

20. Morrison, A., and Cozzarelli, N. R. (1981) Proc. Natl. Acad. Sci. U. S. A. 78, $1416-1420$

21. Orphanides, G., and Maxwell, A. (1994) Nucleic Acids Res. 22, 1567-1575

22. Liu, L. F., and Wang, J. C. (1978) Cell 15, 979-984

23. Lee, M. P., Sander, M., and Hsieh, T. (1989) J. Biol. Chem. 264, 21779-21787

24. Thomsen, B., Bendixen, C., Lund, K., Andersen, A. H., Sorensen, B. S., and Westergaard, O. (1990) J. Mol. Biol. 215, 237-244

25. Ullsperger, C., and Cozzarelli, N. R. (1996) J. Biol. Chem. 271, 31549-31555

26. Stone, M. D., Bryant, Z., Crisona, N. J., Smith, S. B., Vologodskii, A., Bustamante, C., and Cozzarelli, N. R. (2003) Proc. Natl. Acad. Sci. U. S. A. 100, 8654-8659

27. Charvin, G., Bensimon, D., and Croquette, V. (2003) Proc. Natl. Acad. Sci. U. S. A. 100, $9820-9825$

28. Hsieh, T. J., Farh, L., Huang, W. M., and Chan, N. L. (2004) J. Biol. Chem. 279, 55587-55593

29. Morais Cabral, J. H., Jackson, A. P., Smith, C. V., Shikotra, N., Maxwell, A., and Liddington, R. C. (1997) Nature 388, 903-906

30. Berger, J. M. (1998) Biochim. Biophys. Acta 1400, 3-18

31. Wigley, D. B., Davies, G. J., Dodson, E. J., Maxwell, A., and Dodson, G. (1991) Nature 351, 624-629

32. Corbett, K. D., Shultzaberger, R. K., and Berger, J. M. (2004) Proc. Natl. Acad. Sci. U. S. A. 101, 7293-7298

33. Van Duyne, G. D., Standaert, R. F., Karplus, P. A., Schreiber, S. L., and Clardy, J. (1993) J. Mol. Biol. 229, 105-124

34. Otwinowski, Z., and Minor, W. (1997) Methods Enzymol. 276, 307-326

35. Terwilliger, T. C. (2003) Methods Enzymol. 374, 22-37

36. Collaborative Computational Project 4 (1994) Acta Crystallogr. Sect. D Biol. 
Crystallogr. 50, 760-763

37. Cowtan, K. (1994) Joint CCP4 and ESF-EACBM Newsletter on Protein Crystallography, Vol. 31, pp. 34-38

38. Petrey, D., and Honig, B. (2003) Methods Enzymol. 374, 492-509

39. Carson, M. (1991) J. Appl. Crystallogr. 24, 958-961

40. Laskowski, R. J., Macarthur, M. W., Moss, D. S., and Thornton, J. M. (1993) J. Appl. Crystallogr. 26, 283-290

41. Vaguine, A. A., Richelle, J., and Wodak, S. J. (1999) Acta Crystallogr. D55, 191-205

42. Musgrave, D. R., Sandman, K. M., and Reeve, J. N. (1991) Proc. Natl. Acad. Sci. U. S. A. 88, 10397-10401

43. Reece, R. J., and Maxwell, A. (1989) J. Biol. Chem. 264, 19648-19653

44. Ward, D., and Newton, A. (1997) Mol. Microbiol. 26, 897-910

45. Jawad, Z., and Paoli, M. (2002) Structure (Camb.) 10, 447-454
46. Richmond, T. J., and Davey, C. A. (2003) Nature 423, 145-150

47. Knight, S. W., and Samuels, D. S. (1999) EMBO J. 18, 4875-4881

48. Knight, S. W., Kimmel, B. J., Eggers, C. H., and Samuels, D. S. (2000) J. Bacteriol. 182, 2048-2051

49. Swofford, D. L. (2001) Phylogenetic Analysis Using Parsimony (and Other Methods) 4th Ed., Sinauer Associates, Sunderland, MA

50. Wang, J. C. (1998) Q. Rev. Biophys. 31, 107-144

51. Peng, H., and Marians, K. J. (1995) J. Biol. Chem. 270, 25286-25290

52. Cozzarelli, N. R., Boles, T. C., and White, J. H. (1990) in DNA Topology and Its Biological Effects (Cozzarelli, N. R., and Wang, J. C., eds) pp. 139-184, Cold Spring Harbor Laboratory Press, Plainview, NY

53. Crick, F. H. (1976) Proc. Natl. Acad. Sci. U. S. A. 73, 2639-2643 


\section{A Superhelical Spiral in the Escherichia coli DNA Gyrase A C-terminal Domain Imparts Unidirectional Supercoiling Bias}

Alexander J. Ruthenburg, Daina M. Graybosch, John C. Huetsch and Gregory L. Verdine

J. Biol. Chem. 2005, 280:26177-26184.

doi: 10.1074/jbc.M502838200 originally published online May 15, 2005

Access the most updated version of this article at doi: $10.1074 / \mathrm{jbc}$.M502838200

Alerts:

- When this article is cited

- When a correction for this article is posted

Click here to choose from all of JBC's e-mail alerts

Supplemental material:

http://www.jbc.org/content/suppl/2005/05/18/M502838200.DC1

This article cites 51 references, 19 of which can be accessed free at http://www.jbc.org/content/280/28/26177.full.html\#ref-list-1 